\title{
DIGITAL TECHNOLOGY Digital ophthalmoscopy: through a non-specialist lens
}

\author{
Author: Christopher Taylor ${ }^{\mathrm{A}}$
}

The ophthalmoscope has been a mainstay for non-specialist physicians to review a range of disorders affecting patient fundi. Its design has remained unchanged since its inception and while relatively easy to operate, gaining mastery of it can prove challenging. Training is limited by its single-user interface with the lack of attachments to augment its design and therefore facilitate skill acquisition. Documentation is variable depending on the competence of the individual to satisfactorily see enough of the fundi, and the accuracy with which they can describe their findings. Several groups have looked to find adaptions to smartphones in order to develop user-friendly and affordable alternative to the traditional ophthalmoscope. A digital solution has the potential to provide greater versatility by enhancing documentation, improved training pathways and facilitating more efficient care, with options for geographically disparate healthcare settings to access teleophthalmology input.

KEYWORDS: ophthalmoscopy, digital, health informatics

DOI: $10.7861 /$ fhj.2020-0013

\section{Background}

The direct ophthalmoscope is an essential piece of equipment allowing non-ophthalmologist physicians to visualise fundi and investigate a range of pathologies at the patient's bedside. The ophthalmoscope's versatility comes from its ability to provide clear images by correcting for the errors of refraction created from both the patient's and physician's eyes. ${ }^{1,2}$ While simple to use, it can be a challenge to master. ${ }^{1-5}$ This, in part, derives from its fixed overall design which focuses on a single-user interface, with a noticeable absence of attachable components to augment it. 1,3,4

Access to a handily available ophthalmoscope, which has not been misplaced, broken or left without power can prove challenging for day-to-day practice. ${ }^{3}$ There are two common models available in non-ophthalmology areas. The first option is a handheld device with both ophthalmoscope and otoscope head attachments. ${ }^{2}$ The other option is an ophthalmoscope-otoscope unit, which may be either fixed to a wall or mounted on a trolley. This comes with subsequent ergonomic challenges to both the

Author: ${ }^{A}$ acute internal medicine registrar, Northumbria Specialist Emergency Care Hospital, Cramlington, UK patient and the doctor, due to having to mobilise to the unit, or navigate the trolley around the hospital. More formal imaging of the optic disc exists with powerful fundus cameras. ${ }^{5}$ These systems are often larger and more expensive, with access and availability restricted to areas with the greatest need such as ophthalmology or emergency departments. ${ }^{1,4,6}$

Identifying a solution which would capture and record images, while also being portable and low-cost could therefore enhance not only service provision, but also improve documentation and medical training pathways. ${ }^{1,3,4,7}$ It could also facilitate the development of a tele-ophthalmology service, which would allow geographically disparate, or resource-limited healthcare centres to access ophthalmology input directly. ${ }^{8,9}$ This article will therefore review the current progress of digital ophthalmoscopy which, within this article, is primarily referred to as the digitisation of direct ophthalmoscopy.

\section{The digital spectrum of ophthalmoscopes}

Traditional ophthalmoscope-otoscope devices have been the mainstay of fundus imaging for decades in several clinical areas, such as emergency departments, neurology outpatients, general practice and medical admission units. Nevertheless, in an increasingly digitised workplace, these tools are becoming outdated. ${ }^{2}$ Digital fundus cameras and digital indirect ophthalmoscopes are already in circulation but are limited by cost, portability or accessibility. ${ }^{1,5,6}$

One opportunity for future investment could be to adapt smartphones, which can already be found in many healthcare settings, in order to provide a lower-cost, easy-to-use and portable digital solution. ${ }^{4,6,9}$ The basic camera installed on most smartphone models is powerful enough to capture appropriate views. ${ }^{7,9}$ The standard camera applications, however, lack the required finesse to manipulate the focal point or adequate exposure settings to optimise the views necessary for use in direct ophthalmoscopy. ${ }^{4,9-11}$ Technology continues to advance and, with annual updates to phone models and their in-house applications, it will be increasingly easier to implement a digital solution. ${ }^{7}$ Until then, applications exist to improve the versatility of smartphone cameras for use in ophthalmoscopy, typically with an attachable device and lens. ${ }^{7,10}$

The most extensively reviewed smartphone with regards to this is the iPhone. ${ }^{7,9}$ Each model provides slightly different gains in hardware and software. ${ }^{7,9}$ The main limitation in hardware is the lack of proximity of the light-source to the lens, causing issues with glare, exposure and focal length (Fig 1).,12 Using newer iPhone 
a

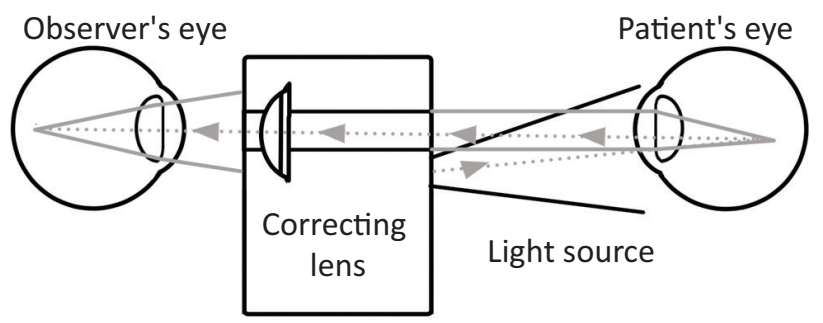

b

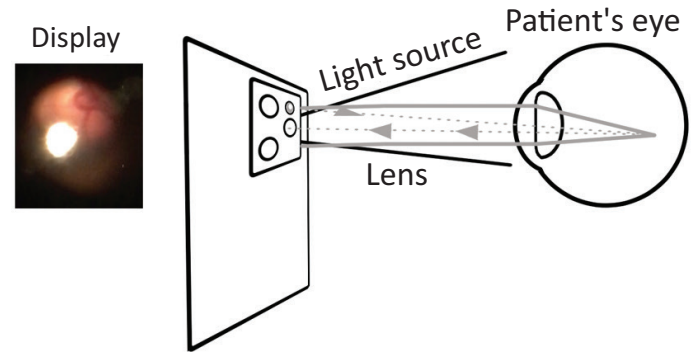

Fig 1. Schemata demonstrating configuration of direct fundoscopy against digital smartphone-based fundoscopy directly showing a problem with glare. a) Direct fundoscopy. b) Digital smartphone fundoscopy.

models, or adapting older models of the iPhone, to resolve this can achieve acceptable quality fundus imaging. These solutions do not yet provide ergonomic or cost-effective options. ${ }^{7,12}$

Several groups have sought to develop applications and attachments to resolve issues with the mobile devices themselves, ranging from do-it-yourself projects, to open-source and highstandard commercially available products. ${ }^{6,9-11,13}$ More creative projects have ranged from video-cameras and nasal endoscopes to readily available recycled equipment from outpatient clinics. ${ }^{6,13}$ Despite these low-cost concepts, the most commonly utilised solution uses a dioptre lens, typically $+20 D$, often with an additional bracket or rig to maintain the focal length. $1,3,4,6,9,11,13$

While this is the most widely reviewed concept, with various companies commercialising this attachment and lens, it comes with additional challenges. Workplaces will be understandably apprehensive to acquire large numbers of easily lost, stolen or damaged lenses and attachments to adapt smartphones. Until smartphones have developed enough to work without these addons, their adoption into routine practice will remain limited, despite evidence demonstrating their benefits. $3,7,10,12$

One significant benefit of digital ophthalmoscopy is the storage of images or videos which could then be saved either directly to the patient electronic healthcare record, or anonymously for teaching and training purposes. ${ }^{3,4,7}$ Easily accessible fundus imaging would provide enhanced documentation by saving time and by increasing accuracy through the minimisation of interpretation or communication errors. ${ }^{3,4,14}$ The confidence of interpretation currently is dependent on the degree of training received, and subsequent frequency and self-rated competence of individual physicians. ${ }^{3}$

\section{Training and competence}

Learning the art of direct ophthalmoscopy takes time and experience. $^{2,3}$ The current process is limited by the design of interface. This means that, during skill acquisition, observers can comment on the device settings, learner's positions, and the limited descriptions provided by the learner about what they can or cannot see. ${ }^{2}$ How well learners can describe what is seen is also a challenge as they are typically taught using whole-fundus photographs, which are then not comparable to the limited, dynamic views seen during direct ophthalmoscopy. ${ }^{4}$ The amount of time dedicated to learning the basic knowledge and techniques required to confidently visualise and comment on a range of ophthalmologic pathologies will vary from person to person, but the time provided during training is often limited. ${ }^{3,4}$

Training can take many forms, such as utilising dummy eyes with indirect ophthalmoscopes, lectures, simulations and specially designed ophthalmoscopes with multiple viewing windows. These are all sub-optimal due to the limitations highlighted above. Also, for ophthalmoscopes with multiple viewing windows, the observer will see weaker images due to less intense light being reflected by the retina., Digital ophthalmoscopy, however, has been proven to build greater competence and confidence in students, optimising their learning within a smaller time-period. 3,4,14

Independence is essential for a lifelong career in healthcare and the current shortfall in training recruitment will likely continue to contribute to inefficiencies in service provision and patient care. ${ }^{1,3,4,7}$ Given the risks associated with failing to visualise the fundus appropriately, training to promote competence and independence is essential to ensure high-quality patient care. ${ }^{5}$ Also, developing skills in taking high-quality images which could be sent to a remotely based ophthalmologist will increase the scope to incorporate multidisciplinary input and would reduce the risks associated with inexperienced non-ophthalmologist interpretation of findings. 4,14

\section{Teleophthalmology}

Telemedicine services are becoming increasingly common across a range of services. ${ }^{8,14}$ Teleophthalmology has yet to become widely adopted, either in the sense of real-time remote consultations, or in the form of "store-and-forward" process of reviewing images and formally reporting these at a convenient time. ${ }^{4,8,14}$ While there remain concerns over the sustainability of these initiatives, examples exist which demonstrate the cost effectiveness and relative ease of innovation in resource limited settings. ${ }^{14}$

Some of the main limitations at present include the digital resilience of healthcare systems, confidence in reporting and the associated risks of reporting. ${ }^{3,4,14,15}$ There are also limitations in the currently available hardware and software itself. These include associated costs, concerns around security and interoperability, as well as the experience of patients and staff with the digital platforms. ${ }^{8,15}$ Counter to these points, the benefits include increased accessibility to ophthalmology input early into the patient journey. This will help to streamline care more efficiently and facilitate appropriate referrals. ${ }^{8}$ With increased contact, there are secondary benefits of enhanced education and improving the knowledge and awareness of best-practice guidelines. ${ }^{4,8}$ In terms of the patient experience, the core benefits are deemed to be convenience, reassurance and personalisation of care within a single encounter. ${ }^{15}$

\section{Conclusion}

While digital ophthalmoscopy remains in its infancy, there is growing momentum of innovation and demonstrations of practical applications to improve service provision. There are clear 
gains to training, documentation and patient care. However, these are currently being limited by lack of investment and early adoption.

A key question with any technology should be whether it provides greater gains to what is being currently delivered or reduces the associated risks. Given the limitations of current equipment, investment in a digital solution should be planned for the near future. While it will never replace more powerful and dedicated fundus imaging, for non-ophthalmologist physicians, the current system remains ineffective and perpetuates the risk for physicians and patients. Patients will therefore continue to receive suboptimal care, facing inefficiencies and inconsistencies, despite best intentions from non-ophthalmologist healthcare professionals. While no clear solution is currently available, in an ever-digitising healthcare service and the growing field of the medical internet-of-things, the main questions now are when and who will be the first to champion digital ophthalmoscopy for nonophthalmologists.

\section{References}

1 Ademola-Popoola DS, Olatunji VA. Retinal imaging with smartphone. Niger J Clin Pract 2017;20:341-5.

2 Timberlake GT, Kennedy M. The direct ophthalmoscope: how it works and how to use it. University of Kansas, 2005.

3 Nagra M, Huntjens B. Smartphone ophthalmoscopy: patient and student practitioner perceptions. Med Syst 2019;44:10.

4 Kim Y, Chao DL. Comparison of smartphone ophthalmoscopy vs conventional direct ophthalmoscopy as a teaching tool for medical students: the COSMOS study. Clin Ophthalmol 2019;13:391-401.

5 Gunasekera CD, Thomas P. High-resolution direct ophthalmoscopy with an unmodified iPhone X. JAMA Ophthalmol 2019;137:212-3.

6 Rathi S, Tsui E, Mehta N, Zahid S, Schuman JS. The current state of teleophthalmology in the United States. Ophthalmology 2017:124:1729-34.
7 Jalil M, Ferenczy SR, Shields CL. iPhone $4 \mathrm{~s}$ and iPhone 5 s imaging of the eye. Ocul Oncol Pathol 2017;3:49-55.

8 Rabbitcreek. Easy iPhone to direct ophthalmoscope conversion. Autodesk, 2013. www.instructables.com/id/Easy-iphone-to-DirectOphthalmoscope-Conversion [Accessed 18 March 2020].

9 Montezuma SR. Smartphone fundoscopy - how to use smartphone to take fundus photographs. American Academy of Ophthalmology, 2018. https://eyewiki.aao.org/Smartphone_ Funduscopy-How_to_use_smartphone_to_take_fundus_photographs [Accessed 18 March 2020].

10 Myung D, Jais A, Lingmin H, Blumenkranz MS, Chang RT. 3D printed smartphone indirect lens adapter for rapid, high quality retinal imaging. J Mob Technol Med 2014;3:9-15.

11 Chandrakanth P, Ravichandran R, Subhashini M. Trash to treasure Retcam. Indian J Ophthalmol 2019;67:541-4.

12 Shanmugan MP, Mishra DKC, Madhukumar R. Unconventional techniques of fundus imaging: A review. Indian J Ophthalmol 2015;63:582-5.

13 Bruce BB, Biousse V, Newman NJ. Nonmydriatic ocular fundus photography in neurologic emergencies. JAMA Neurol 2015;72:455-9.

14 Hong K, Collon S, Chang D et al. Teleophthalmology through handheld mobile devices: a pilot study in rural Nepal. J Mob Technol Med 2019;8:10.7309/jmtm.8.1.1.

15 Ramchandran RS, Yilmaz S, Greaux E, Dozier A. Patient perceived value of teleophthalmology in an urban, low income US population with diabetes. PLoS One 2020;15:e0225300.

Address for correspondence: Dr Christopher Taylor, Northumbria Specialist Emergency Care Hospital, Northumbria Healthcare NHS Foundation Trust, Northumbria Way, Cramlington NE29 8NH, UK.

Email: chris.taylor28@nhs.net

Twitter: @gingernutter88 